\title{
Gender-specific 11-dehydro-thromboxane B2 levels in acute coronary syndrome and its association with clinical outcomes
}

\author{
Sheetal Chauhan ${ }^{1}$, Ajit Singh ${ }^{2}$, Yeshwanth Rao Karkala ${ }^{3 *}$, Tom Devasia ${ }^{2}$, Hashir Kareem ${ }^{4}$, Deepak Uppunda², Prasad Narayana \\ Shetty ${ }^{2}$, Ganesh Paramasivam ${ }^{2}$ \\ ${ }^{1}$ Department of Pharmacology, Melaka Manipal Medical College, Manipal Academy of Higher Education, Manipal, India. \\ ${ }^{2}$ Department of Cardiology, Kasturba Medical College, Manipal Academy of Higher Education, Manipal, India. \\ ${ }^{3}$ Department of Pharmacology and Ethics, Saint James School of Medicine, Anguilla (BWI), UK. \\ ${ }^{4}$ Department of Cardiology, Kerala Institute of Medical Sciences, Trivandrum, India.
}

\begin{tabular}{l}
\hline ARTICLE INFO \\
\hline Received on: 04/05/2020 \\
Accepted on: 29/09/2020 \\
Available online: 05/11/2020 \\
\\
Key words: \\
Major adverse cardiac \\
events, cardiac death, aspirin, \\
percutaneous transluminal \\
coronary angioplasty, ELISA \\
assay.
\end{tabular}

\begin{abstract}
Despite knowing that females are at a higher risk of major adverse cardiac events (MACE) and poor prognosis after percutaneous transluminal coronary angioplasty (PTCA), there are very few studies focusing on urinary 11-dehyrdothromboxane B2 (dh-TxB2) concentration (for aspirin non-responsiveness) differences among males and females. The present prospective observational study aimed to evaluate the dh-TxB2 levels and associate them with clinical outcomes in males and females with acute coronary syndrome (ACS) after PTCA. The dh-TxB2 ELISA assay was carried out twice, at baseline and 6 months follow-up, and outcomes were assessed for 1 year. The average cut-off value of dh-TxB2 was $\geq 1,496 \mathrm{pg} / \mathrm{mg}$ creatinine by the receiver operating characteristic curve estimation. A total of 192 patients (140 men and 52 women) were enrolled. At baseline, 17 (12.1\%) males and 7 (13.5\%) females had high dh-TxB2 levels, whereas at 6 months, $22(15.9 \%)$ males and 4 (7.8\%) females showed high dh-TxB2 levels. The concentration of dh-TxB2 was higher in females than males at baseline. Composite MACE was not different between genders significantly even when the dh-TxB2 levels were high. Women experienced higher MACE incidences than men $(13.5 \%$ vs. $9.3 \%)$.
\end{abstract}

\section{INTRODUCTION}

Acute coronary syndrome (ACS) is caused by coronary atherosclerosis rupture, platelet activation, aggregation, and thrombosis and is significantly associated with morbidity and mortality. Antiplatelet therapy, especially aspirin, is the most important part of pharmacotherapy to lessen the risk of future cardiovascular (CV) events (Badimon et al., 2012; Smith et al., 2015). It acts through acetylating platelet cyclooxygenase-1 (COX-1) irreversibly, inhibiting the formation of thromboxane A2 (TxA2). The end metabolite 11-dehyrdo-thromboxane B2 (dhTxB2) is a stable biomarker of systemic TxA2 biosynthesis and

\section{"Corresponding Author}

Yeshwanth Rao Karkala, Department of Pharmacology and Ethics, Saint James School of Medicine, Anguilla (BWI), UK.

E-mail:yrao@mail.sjsm.org is excreted in the urine, which gives a reliable estimation of the total in vivo production of TxA2 (Liu et al., 2018; McCullough et al., 2017). Prior studies have showed that its level had been elevated in several atherothrombotic Cardiovascular Diseases (CVDs) (Gonçalves et al., 2011; Wang et al., 2018). Percutaneous transluminal coronary angioplasty (PTCA) is one of the preferred treatments for most patients presenting with ACS worldwide (Badimon et al., 2012; Smith et al., 2015). However, within the first year after the procedure, the risk of recurrent $\mathrm{CV}$ events (death, myocardial infarction (MI), and repeat revascularization) is high (Johansson et al., 2017; Liu et al., 2018).

Multiple studies have pointed out the gender differences in pathophysiological and clinical expressions of atherosclerosis. The female gender has been associated with poorer prognosis after coronary revascularization and has a high risk of death and MI (Josiah and Farshid, 2019; Guo et al., 2018). Some studies suggest that the female gender itself is an independent risk factor for the outcomes of $\mathrm{CV}$ and have persistent gender differences in 
outcomes after angioplasty, while other studies have demonstrated equal probabilities of occurrence of events in male and females (Kurlansky et al., 2017; Pendyala et al., 2013; Rao et al., 2019).

Earlier studies have focused on urinary thromboxane, demonstrating that the perpetual thromboxane level in CVD patients was high and had an increased risk of atherothrombosis and death, although they were on aspirin therapy (Gabrielsen et al., 2010; Johansson et al., 2017; McCullough et al., 2017). Despite knowing that females are at a higher risk of major adverse cardiac events (MACE), there are very few studies focusing on urinary dh-TxB2 concentration differences among males and females. The aim of the present study is to investigate the gender-specific dhTxB2 level and its association with MACE after PTCA in ACS patients.

\section{METHODS}

\section{Information disclosure}

The study protocol was approved by the Institutional Ethics Committee at Manipal Academy of Higher Education in India. All the subjects provided written informed consent for participating and sample collection under the present study. The approval statement was issued in January 2018 with the trial registration number CTRI/2018/01/011578 (Clinical Trial Registry - India).

\section{Study design and population}

This is a prospective observational study that recruited a cohort of patients who underwent percutaneous coronary intervention (PCI) with drug-eluting stents (DES) at a tertiary care university hospital located in South India. Patients were directly presented or referred from all associated primary care hospitals and clinics between May 2017 and April 2018. Recruited patients were followed-up for 1 year following index hospitalization (Fig. 1).

The study subjects were considered eligible for inclusion if they were aged $\geq 18$ years, had been diagnosed with ACS for the first time, and successfully underwent PCI with at least one DES. They would receive dual antiplatelet therapy (aspirin along with a P2Y12 inhibitor) loading doses for the first time.

The main exclusion criteria were oral or parenteral anticoagulation, previous treatment with anticoagulant, nonsteroid anti-inflammatory drugs in the first months, psychiatric illness on medications, pregnancy, cardiogenic shock on presentation or during hospitalization, history of coronary artery disease (CAD), severe valvular heart disease, active malignancy or chronic inflammatory conditions, hemorrhagic diathesis, liver cirrhosis, hematological or neoplastic carcinoma, terminal malignancy patients with a life expectancy of less than 1 year, severe liver disease, and patients who refused to participate in the study. Other exclusion criteria were hemoglobin $<10 \mathrm{~g} / \mathrm{dl}$ (for both males and females), platelets $<100,000 / \mathrm{mm}^{3}$ or $>500,000 /$ $\mathrm{mm}^{3}$, serum creatinine $>1.2 \mathrm{mg} / \mathrm{dl}$, heart failure, ejection fraction (EF) $<40 \%$ by echocardiography, and current use of inotropes or vasopressors.

All screened patients were administered aspirin $325 \mathrm{mg}$ with ticagrelor $180 \mathrm{mg}$ or clopidogrel $300 \mathrm{mg}$ as the loading dose. At hospital discharge, patients were prescribed aspirin ( 75 or 150

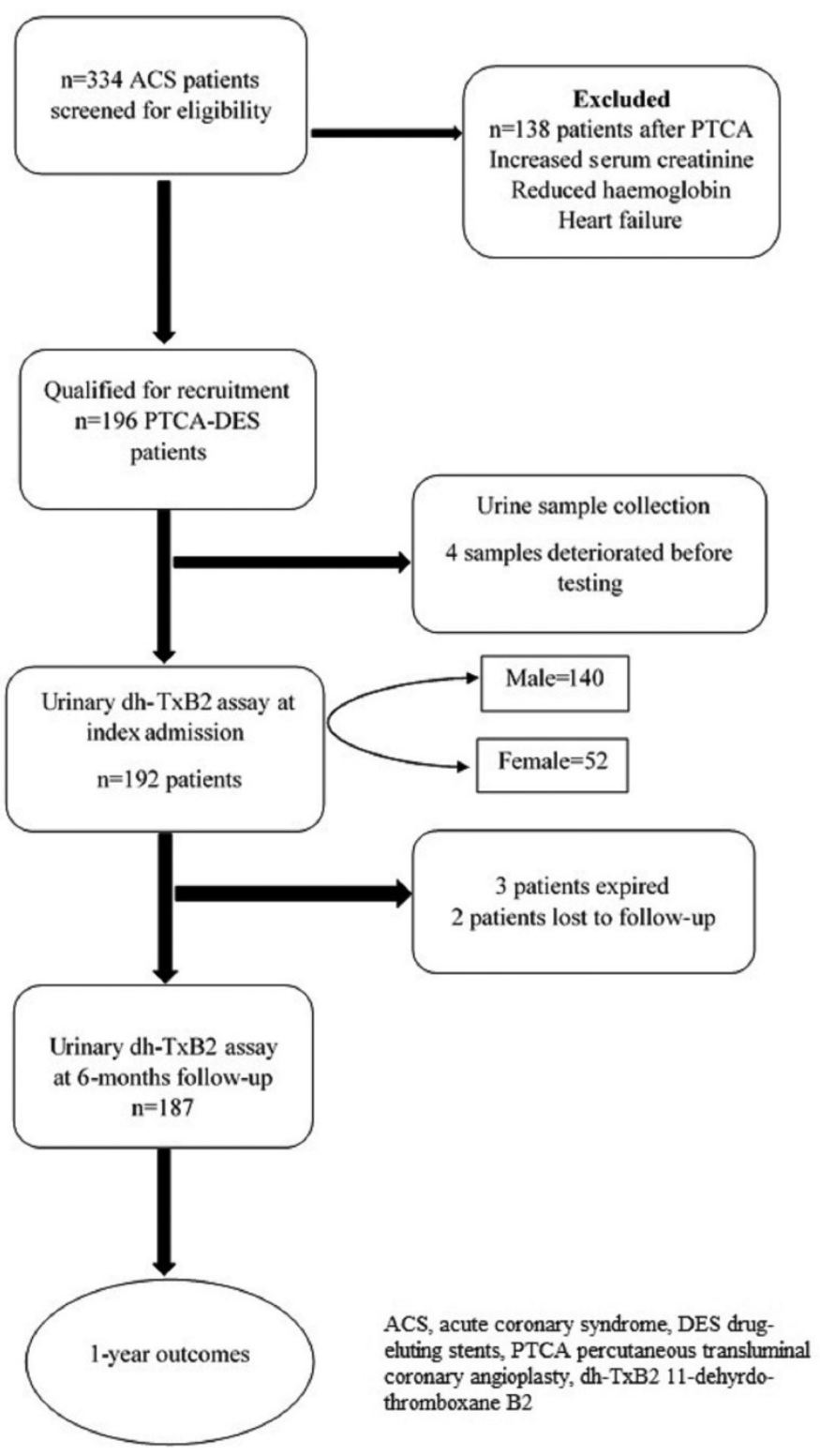

Figure 1. Study flow chart.

$\mathrm{mg}$ ) with ticagrelor $180 \mathrm{mg}$ or clopidogrel (75 or $150 \mathrm{mg}$ ) daily and were instructed to take medicines without fail till the next follow-up visit or unless directed otherwise by a local physician.

\section{Sample collection and processing}

For all enrolled patients in the study, blood samples and urine samples were obtained. Blood samples were collected by clean venipuncture to carry out standard laboratory tests at index hospitalization. For dh-TxB2 measurement, the first morning urine sample was collected within 24 hours of angioplasty and stored immediately at $-80^{\circ} \mathrm{C}$ pending analysis. The samples were defrosted at room temperature and further centrifuged at $1,000 \mathrm{~g}$ for 15 minutes and then the supernatant was collected for analysis. The test was carried out within 3 months of sample collection with the colorimetric method. Furthermore, a urine sample was collected at 6 months follow-up for repeat dh-TxB2 assay. 


\section{Laboratory studies}

For all the recruited subjects, systemic TxA2 was evaluated by measuring concentrations of metabolite (stable) dh-TxB2 in the urine via a commercially available ELISA assay (UTxB2: assay \#519510, Cayman Chemical, Ann Arbor, $\mathrm{MI}$ ) according to manufacturer's instructions. TxA2 is an unstable metabolite which is rapidly converted into more stable (chemically) and relatively biologically inactive metabolites (TxB2) and is excreted in the urine in the form of dh-TxB2. Thus, urinary dh-TxB2 serves as a reliable substitute to measure total TxA2 formation in vivo (Gonçalves et al., 2011; Liu et al., 2018; Vasudevan et al., 2018). The dh-TxB2 concentrations were normalized in equilibrium to urinary creatinine $(\mathrm{Cr})$ concentrations to avoid inter-individual differences in urine dilution. The results were expressed as a ratio, in picograms of dh-TxB2 per milligram of urine $\mathrm{Cr}$ ( $\mathrm{pg} / \mathrm{mg} \mathrm{Cr})$.

\section{Baseline characteristics}

Socio-demographics characteristics, data on comorbid conditions, history of presenting complaints, medical history, and medication history were collected when patients were enrolled in the study. All the crucial aspects of coronary interventions and CV symptoms were assessed. Electrocardiography, echocardiography, coronary angiogram, and angioplasty were carried out, and the results were noted for analysis.

\section{Follow-up}

All the recruited patients were followed-up for 1 year after enrollment in the study. Patients' visits were scheduled in our outpatient department, at 1, 6, and 12 months intervals after first-time PCI-DES procedure. At every follow-up, the occurrence of MACE was collected. Other data of clinical parameters and Dual Antiplatelet Therapy (DAPT) utilization and medication switchover were documented. At 6 months, again urine samples were collected for dh-TxB2 level assessment, and the test was carried out in the same manner as that mentioned at baseline.

\section{Study's clinical outcomes}

The primary outcome of the study consisted of MACE, "a composite of MI [unstable angina, non-ST segment elevation MI (NSTEMI), ST-segment elevation MI (STEMI)], stroke, target lesion revascularization, target vessel revascularization, and death due to any cause."

The secondary outcome included heart failure, and it was not included in the primary outcomes. If the occurrence of MACE was more than once in a patient (e.g., recurrent MI and death during follow-up), only the first event was considered. Three cardiologists who were blinded to dh-TxB2 results independently adjudged all events.

\section{Statistical analysis}

Baseline characteristics were presented as percentage or proportions for categorical variables, and continuous variables were reported as mean $\pm \mathrm{SD}$ or median, depending on their distribution. Proportions were compared using the chi-square test and Fisher's exact test. Continuous variables were analyzed by Student's $t$-test for normally distributed data. The impact of covariates, such as age, smoking, diabetes, hypertension, dyslipidemia, the number of coronary arteries involved, multivessel disease, and dh-TxB2 on MACE, was analyzed with Cox's proportional hazards regression model. The correlations between variables were analyzed using Pearson's correlation. The time origins for the survival analyses were set at the first time of angioplasty to the time of the first occurrence of a CV event. Univariate logistic regression analysis was carried out to evaluate the variables associated with MACE between genders. Finally, optimized multivariable Cox proportional hazard models were used by selecting the variables based on univariate logistic results $(p<0.20)$. Kaplan-Meier's survival plots were constructed for time-to-event analysis at 1 year for the association of dh-TxB2 levels between genders. A $p$-value $<0.05$ was considered to be significant. All statistical analyses were carried out using Statistical Package for the Social Sciences 22.0 software. To evaluate the ability of dh-TxB2 in the prediction of cumulative MACE, receiver operating characteristic (ROC) curve analysis was conducted. We calculated the area under the curve and based on the best sensitivity/specificity results the optimal threshold value of dh-TxB2 was obtained for diagnostic purposes (Vasudevan et al., 2018).

\section{RESULTS}

\section{Clinical characteristics of the study population}

A total of 192 ACS patients after successful PTCA on index hospitalization were found to be eligible for the study. The baseline clinical characteristics, socio-demographics, and medication details of the study population are shown for both genders in Table 1. Dual antiplatelet medication utilization rate was consistent with current clinical practice guidelines, and no difference was observed between genders (Table 1).

\section{dh-TxB2 levels}

We tested thromboxane metabolite for 192 patients within 24 hours after PTCA at index admission and for 187 patients at 6 months (two patients were lost to follow-up and three patients expired before completing 6 months follow-up). An optimal cutoff point revealed an average threshold of $1,495.8 \mathrm{pg} / \mathrm{mg} \mathrm{Cr}$ with the help of the ROC curve, which was near to the reference cutoff value (1,500 pg/mg Cr) (Fig. 2). At baseline, 12.5\% (n, 24) of patients showed a high dh-TxB2 level than the specified cutoff value, and at 6 months follow-up, 13.9\% (n, 26) of patients showed a high dh-TxB2 level. At baseline, 17 (12.1\%) males and $7(13.5 \%)$ females had high dh-TxB2 levels, whereas at 6 months, $22(15.9 \%)$ males and $4(7.8 \%)$ females showed high dh-TxB2 levels. A significant difference between dh-TxB2 concentrations in males and females was noted at baseline but not at 6 months [Baseline: 555.7 vs. 889.2 pg/mg Cr. $(p=0.01)$; 6 months: 561.3 vs. $756.8 \mathrm{pg} / \mathrm{mg} \mathrm{Cr}(p=0.089)$ (males vs. females, respectively)] (Fig. 3).

\section{Gender and outcomes}

Twenty patients $(10.4 \%)$ experienced MACE at least once during the 1-year follow-up period. Ischemic heart failure and revascularization were comparable between genders ( $p$-value $<0.05$ ), but cumulative MACE was not significantly comparable (Table 2). 
Table 1. Baseline characteristics in both genders.

\begin{tabular}{|c|c|c|c|}
\hline Variables & Male $(n=140)$ & Female $(n=52)$ & $p$-value \\
\hline \multicolumn{4}{|l|}{ Clinical characteristics } \\
\hline Age & $57.3 \pm 11.2$ & $62.6 \pm 9.3$ & 0.003 \\
\hline Smoking & $51(36.4 \%)$ & $12(23.1 \%)$ & 0.006 \\
\hline Hypertension & $73(52.1 \%)$ & $33(63.5 \%)$ & 0.107 \\
\hline Diabetes mellitus & $64(45.7 \%)$ & $20(38.5 \%)$ & 0.231 \\
\hline Dyslipidemia $^{\mathrm{a}}$ & $68(48.6 \%)$ & $28(56.0 \%)$ & 0.078 \\
\hline Body mass index & $23.4 \pm 2.8$ & $24.1 \pm 3.7$ & 0.112 \\
\hline $\mathrm{EF}$ & $55.8 \pm 8.0$ & $58.3 \pm 8.4$ & 0.068 \\
\hline \multicolumn{4}{|l|}{ Disease type } \\
\hline Unstable angina & $31(22.1 \%)$ & $10(19.2 \%)$ & \\
\hline STEMI & $94(67.1 \%)$ & $30(57.7 \%)$ & 0.091 \\
\hline NSTEMI & $15(10.7 \%)$ & $12(23.1 \%)$ & \\
\hline Single vessel disease & $52(37.1 \%)$ & $19(36.5 \%)$ & \\
\hline Double vessel disease & $54(38.6 \%)$ & $22(42.3 \%)$ & 0.862 \\
\hline Multivessel disease & $34(24.3 \%)$ & $11(21.2 \%)$ & \\
\hline Involvement of LAD & $104(74.3 \%)$ & $39(75 \%)$ & 0.540 \\
\hline \multicolumn{4}{|l|}{ Platelet indices } \\
\hline Platelet count & $249.5(114.0,609.0)$ & $286.0(138.0,474.0)$ & 0.113 \\
\hline Platelet distribution width & $16.76 \pm 0.66$ & $16.75 \pm 0.58$ & 0.078 \\
\hline Mean platelet volume & $8.26 \pm 0.92$ & $8.29 \pm 0.82$ & 0.783 \\
\hline \multicolumn{4}{|c|}{ Medications during index hospitalization } \\
\hline Aspirin $^{\mathrm{b}}$ & $139(99.3 \%)$ & $51(98.1 \%)$ & 0.469 \\
\hline Ticagrelor & $126(90.0 \%)$ & $47(90.4 \%)$ & 0.589 \\
\hline Clopidogrel & $14(10.0 \%)$ & $4(7.7 \%)$ & 0.431 \\
\hline Statins & $138(98.6 \%)$ & $52(100.0 \%)$ & 0.385 \\
\hline Beta blockers & $55(39.3 \%)$ & $21(40.4 \%)$ & 0.509 \\
\hline ACE inhibitors/ARBs & $51(36.4 \%)$ & $19(36.5 \%)$ & 0.469 \\
\hline
\end{tabular}

STEMI = ST-elevated myocardial infarction; NSTEMI = non-ST-elevated myocardial infarction; RCA $=$ right coronary artery; $\mathrm{LAD}=$ left anterior descending artery; $\mathrm{LCx}=$ left circumflex coronary artery; $\mathrm{ACE}=$ angiotensin-converting enzyme; $\mathrm{ARB}=$ angiotensin receptor blockers.

aDslipidemia is defined as total cholesterol $>250 \mathrm{mg} / \mathrm{dl}$, low density lipids (LDL) $>130 \mathrm{mg} / \mathrm{dl}$, and high density lipids $(\mathrm{HDL})<40 \mathrm{mg} / \mathrm{dl}(<50 \mathrm{mg} / \mathrm{dl}$ for women) in the fasting state.

${ }^{b}$ Loading dose of antiplatelet drugs was administered to all 192 patients (aspirin loading dose $=325 \mathrm{mg}$; clopidogrel loading dose $=300 \mathrm{mg}$, and ticagrelor $=180 \mathrm{mg}$ ).

\section{Gender, dh-TxB2, and outcomes}

Male patients who had high dh-TxB2 at baseline developed significantly comparable MACE events against the males with normal dh-TxB2 levels $(41.2 \%$ vs. $5.7 \%$, $p$-value $<0.001)$. In female patients also, the difference in MACE with high dh-TxB2 levels manifested against low dh-TxB2, but it was not statistically significant $(14.3 \%$ vs. $11.1 \%, p$-value $=0.600)$.

Multivessel disease and high dh-TxB2 levels were found to be the best predictors of 1-year cumulative MACE in male patients by univariate and multivariate analysis. While in females, high dh-TxB2 was the only factor associated with 1-year cumulative MACE (Table 3).

Kaplan-Meier's curve by survival analysis demonstrated an increased risk of the early development of MACE in men with high dh-TxB2 in comparison to men with normal dh-TxB2 levels (239 vs. 352 days; log-rank $p$-value <0.001). In women, there was no significant difference $(270$ vs. 365 days; log-rank $p$-value $=$ 0.111) (Fig. 4).

\section{DISCUSSION}

From this study, comparing dh-TxB2 levels and outcomes in patients on aspirin therapy following PTCA, the following major findings were obtained:

A significant proportion of patients had high dh-TxB2 levels at baseline, indicating aspirin non-responsiveness.

The mean baseline dh-TxB2 concentration was higher in females compared to males.

A baseline dh-TxB2 level $(\geq 1,496 \mathrm{pg} / \mathrm{mg} \mathrm{Cr})$ in males predicted 1-year MACE but not in females.

Gender-based outcome studies in ACS patients after PCI reported vague results. These studies have shown worse in-hospital and long-term clinical outcomes among females compared with males, especially higher rates of recurrent events during long-term follow-up (Josiah and Farshid, 2019; Kurlansky et al., 2017; Rao et al., 2019; Vasudevan et al., 2018). The role of thromboxane, especially in males and females, has not been investigated. In our study cohort, women exhibited a high 


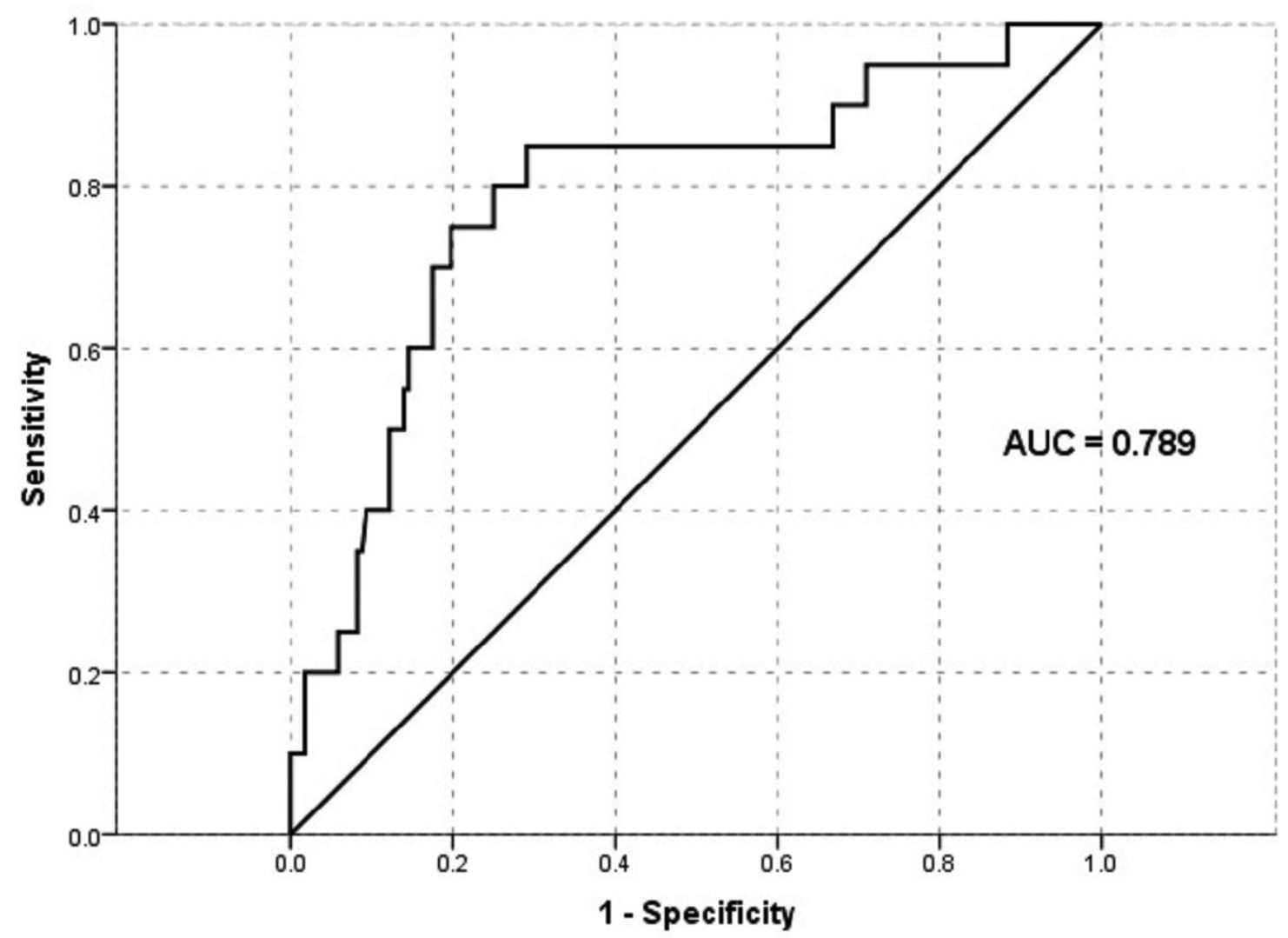

Figure 2. ROC to estimate the cut-off value of dh-TxB2 at baseline with 1-year cumulative MACE.

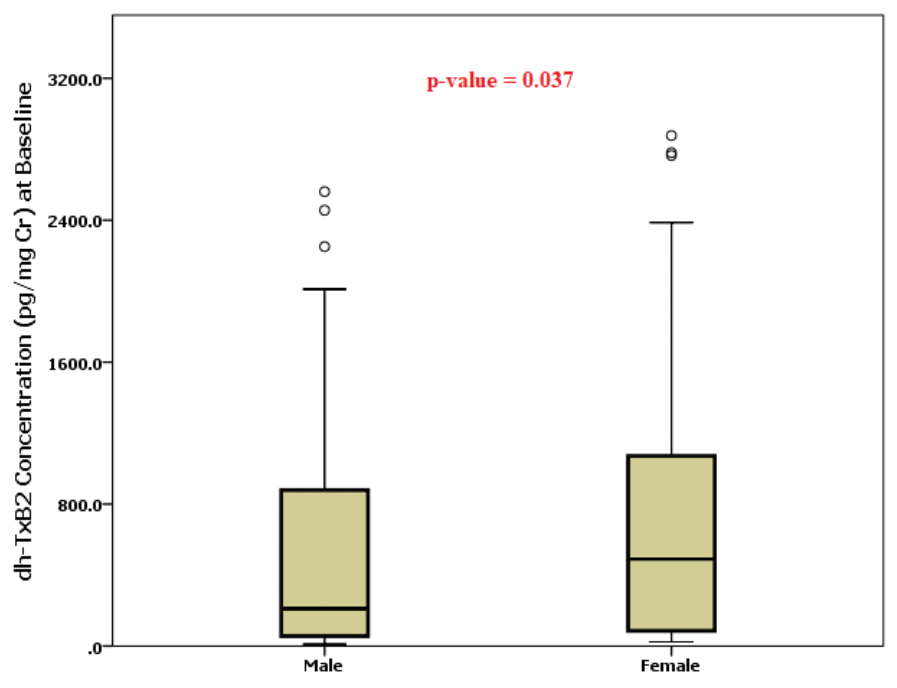

A. Baseline

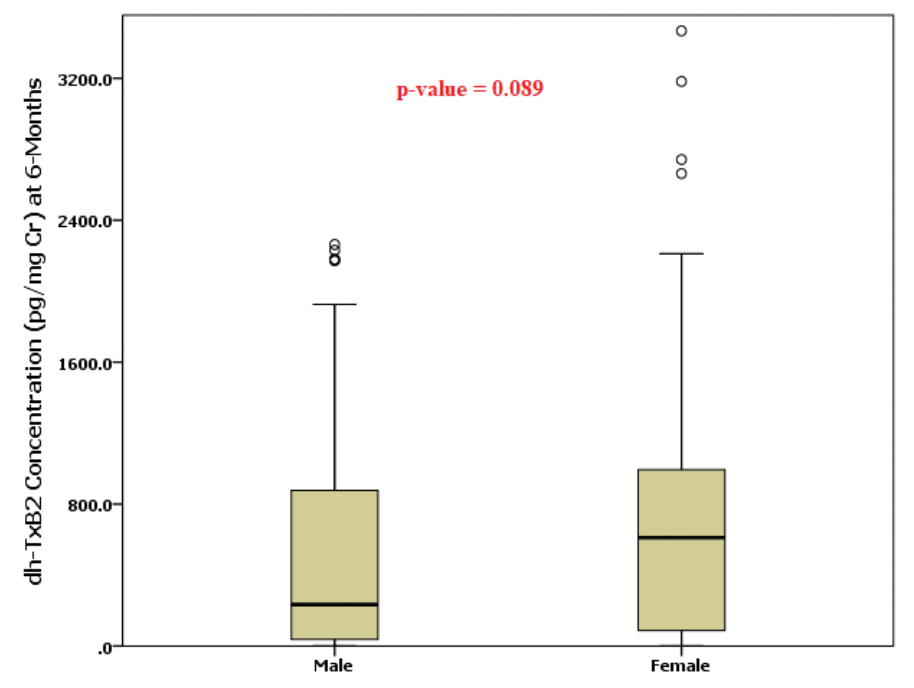

B. 6-Months

Figure 3. Box plots showing dh-TxB2 concentration differences between men and women at $[\mathrm{A}]$ baseline and [B] 6 months.

concentration of thromboxane metabolite compared to men at both time points, but overall concentration was decreased from baseline to 6 months follow-up in both genders. In subgroup analysis, we observed that dh-TxB2 levels during baseline were higher in patients who experienced MACE at 1 year compared to those who did not. These findings are in line with previous studies. Szczeklik et al. (2016) demonstrated that the mean dh-TxB2 measured at baseline during acute MI was higher on admission when compared to 1-month and 12 months and patients who developed MACE showed a higher mean concentration of dh-TxB2 compared to those who did not develop MACE. In reduction in graft occlusion rates study, researchers also observed 
that thromboxane generation was decreased subsequently from baseline to 6 months after coronary artery bypass graft surgery (CABG) (Kakouros et al., 2017).

Contemporary studies are showing that, in patients with CVDs, only platelet is not responsible for thromboxane generation; other inflammatory mediators are responsible for it (Cipollone et al., 2000; Kakouros et al., 2016). Numerous factors are responsible immediately after MI and intervention/surgery in cardiac patients. In the acute phase, myocardial injury induces intense physiologic stress and inflammation; platelets get over-

Table 2. Composite outcomes.

\begin{tabular}{lcccc}
\hline Variables & Male $(\mathrm{n}, 140)$ & Female $(\mathrm{n}, 52)$ & Total $(\mathrm{n}, 192)$ & $\boldsymbol{p}$-value \\
\hline Cumulative MACE & $18(9.3 \%)$ & $11(13.5 \%)$ & $29(10.4 \%)$ & 0.469 \\
All cause death & $3(2.1 \%)$ & $3(5.7 \%)$ & $6(3.1 \%)$ & 0.071 \\
Non-fatal MI & $6(4.3 \%)$ & $3(5.7 \%)$ & $9(4.7 \%)$ & 0.671 \\
Revascularization & $4(2.9 \%)$ & $1(1.9 \%)$ & $5(2.6 \%)$ & $<0.001$ \\
Ischemic heart failure & $5(3.6 \%)$ & $4(7.7 \%)$ & $9(4.7 \%)$ & 0.038 \\
\hline
\end{tabular}

$\mathrm{CV}=$ cardiovascular; $\mathrm{MI}=$ myocardial infarction $; \mathrm{MACE}=$ major cardiac adverse events. activated and are released in high amounts. Sometimes, newly formed platelets increase thromboxane synthesis by retaining COX-2 activity along with COX-1 expression. Besides this, in acute inflammatory conditions, thromboxane is being synthesized from inflammatory cells (monocytes and macrophages) and endothelial cells. Mostly, these inflammatory cells produce thromboxane via the COX-2 pathway (Ames et al., 2012; Gonçalves et al., 2011; Szczeklik et al., 2016; Wang et al., 2018). In our cohort, later at 6 months, when the subjects would have likely returned to their physiologic baseline, thromboxane release had decreased. It was strongly correlated with previous evidence from other studies of increased oxidative stress and affected by age and sex, similar to the studies of patients with stable CVD or diabetes mellitus or CABG receiving aspirin therapy (Ames et al., 2012; Kakouros et al., 2016).

This is the first study designed to identify the specific dh-TxB2 concentration between male and female post-PTCA and is prescribed with dual antiplatelet therapy. Previous studies have shown that female patients generate more thromboxane compared to males. A study investigated 100 diabetic patients and healthy controls and found that females exhibited a mean baseline dh-

Table 3. Univariate and multivariate analysis for correlates of 1-year major cardiac adverse events in males and females.

\begin{tabular}{|c|c|c|c|c|c|c|c|c|}
\hline \multirow{3}{*}{ Variables } & \multicolumn{4}{|c|}{ Male (n, 140) } & \multicolumn{4}{|c|}{ Female (n, 52) } \\
\hline & \multicolumn{2}{|c|}{ Univariate analysis } & \multicolumn{2}{|c|}{ Multivariate analysis } & \multicolumn{2}{|c|}{ Univariate analysis } & \multicolumn{2}{|c|}{ Multivariate analysis } \\
\hline & OR & $p$-value & OR & $p$-value & OR & $p$-value & OR & $p$-value \\
\hline Hypertension & $2.42(0.76,7.74)$ & 0.134 & $1.33(0.37,4.77)$ & 0.661 & $1.18(0.22,6.44)$ & 0.849 & $0.74(0.12,4.44)$ & 0.741 \\
\hline Diabetes & $2.14(0.72,6.39)$ & 0.172 & $1.15(0.34,3.86)$ & 0.818 & $1.75(0.35,8.72)$ & 0.490 & $2.30(0.30,17.59)$ & 0.421 \\
\hline SVD vs. MVD & $6.25(2.09,18.67)$ & 0.001 & $10.53(2.67,41.60)$ & 0.001 & $1.91(0.35,10.46)$ & 0.454 & $3.19(0.41,24.88)$ & 0.269 \\
\hline LAD involvement & $4.36(1.09,17.37)$ & 0.037 & $1.43(0.33,6.15)$ & 0.628 & $0.39(0.02,9.31)$ & 0.565 & $0.21(0.02,2.11)$ & 0.186 \\
\hline RCA involvement & $2.25(0.71,7.18)$ & 0.170 & $1.12(0.29,4.22)$ & 0.867 & $0.79(0.04,15.52)$ & 0.880 & $0.21(0.02,1.86)$ & 0.159 \\
\hline dh-TxB2 at baseline ${ }^{a}$ & $8.67(2.99,25.09)$ & $<0.001$ & $15.27(4.03,57.82)$ & $<0.001$ & $6.89(1.26,37.72)$ & 0.026 & $20.78(1.95,221.55)$ & 0.012 \\
\hline
\end{tabular}

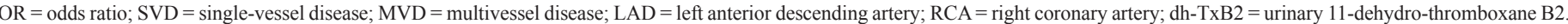
${ }^{a}$ High dh-TxB2 levels ( $\geq 1,496 \mathrm{pg} / \mathrm{ml}$ serum creatinine).

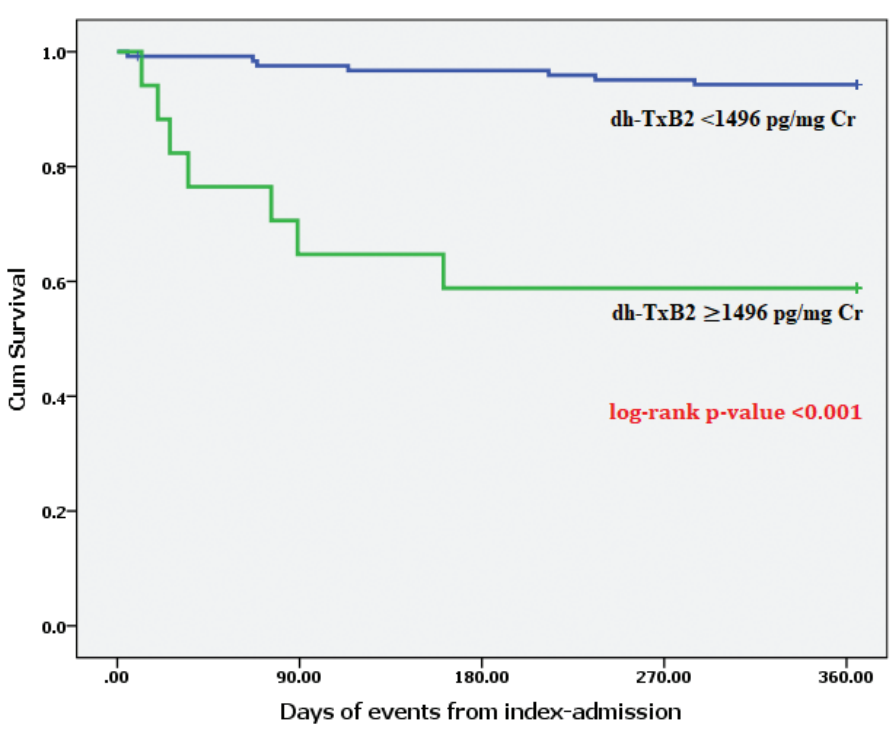

A. Males

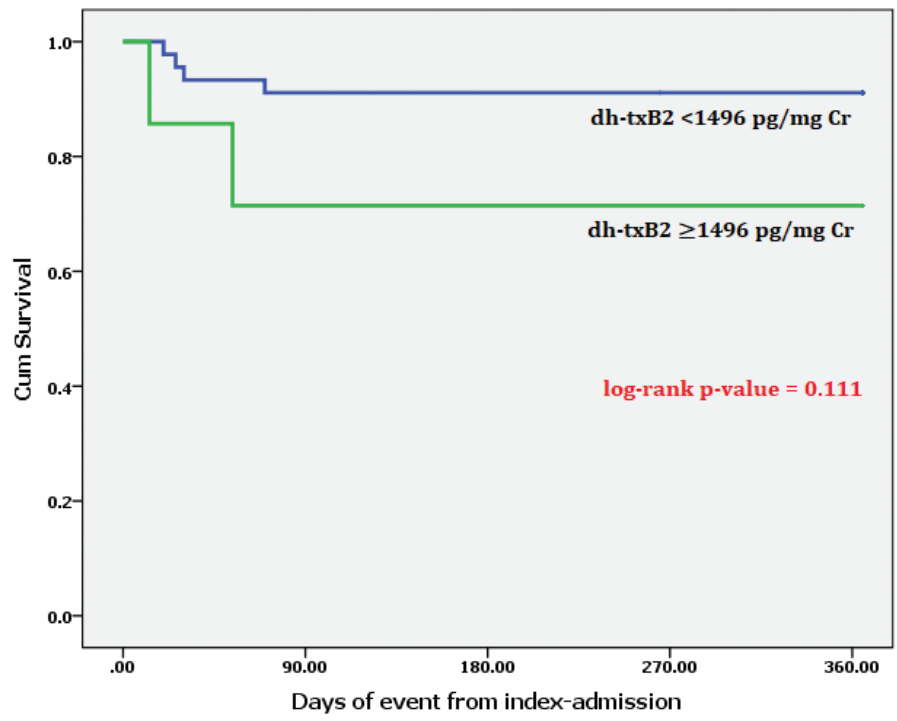

B. Females

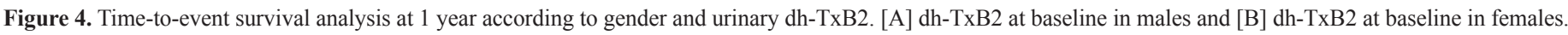


TxB2 50.9\% higher than that of males (5,902 vs. 2,998 pg/mg, $p=$ $0.0004)$. Similarly, in our study, females showed a higher dh-TxB2 level at baseline as well as at 6 months follow-up when compared to post-PTCA males (Lopez et al., 2014).

Prior studies have reported that female patients with ACS undergoing PCI differ from male participants in some baseline characteristics; females are more frequently older with a high prevalence of coronary risk factors, such as diabetes mellitus, hypertension, renal insufficiency, higher prevalence of advanced heart failure, smaller access, lower estimated glomerular filtration rate, higher CAD risk profile, and high rate of bleeding complications as compared with males (Guo et al., 2018; Johansson et al., 2017; Rao et al., 2019). The socioeconomic factor was also linked to a higher risk of recurrent events, i.e., females may delay hospital visits and revascularization therapy compare to males (Lopez et al., 2014; Kareem et al., 2018). In our present study, although males tended to have more diabetes mellitus and were younger than female patients, older females were at a higher risk of all-cause mortality. While males were associated with a higher rate of revascularization compared to females $(p<0.001)$, it has been observed that there is around 10-20 years delay in the onset of CVD in females and carries more CV risks and comorbidities compared to males; it can be considered as a possible cause by explaining a higher event rate in the female gender (Liu et al., 2018; Rao et al., 2019). In our study, patients who died had confounding factors like left anterior descending artery (LAD) involvement multivessel disease and more than one comorbidity. On the contrary, females were older than 65 years and males were mostly 50 years old. Also, females who died had high urinary thromboxane levels compared to male patients. McCullough et al. (2017) observed in their stable CAD patients that the risk of mortality increased in proportion with the rising concentrations of dh-TxB2 after adjusting for age, worsening renal function, and other comorbidities. Even the heart outcomes prevention evaluation study showed that patients categorized in the upper quartile of dh-TxB2 had a 1.8 times higher risk of $\mathrm{CV}$ death compared with those in the lower quartile (Eikelboom et al., 2002). The CHARISMA trial also demonstrated that increased dh-TxB2 levels in patients who were on aspirin therapy had been linked to a higher risk of adverse cardiac events, such as stroke, MI, and CV death (Eikelboom et al., 2008). In line with these studies, we also found that the risk of MACE increased linearly with increasing concentrations of dh-TxB2. High dh-TxB2 levels in males at baseline $(p<0.001)$ were significantly associated with 1 -year cumulative MACE, but in females, it was weakly associated at 6 months $(p=0.026)$.

\section{Limitations}

Our study has some potential limitations; first, this is a single-center study with a limited number of subjects. Second, we were unable to evaluate some other factors which could influence dh-TxB2 concentrations, like the patient's genetic variability of thromboxane production or thromboxane receptor synthesis, thromboxane synthesis other than platelets, and its turnover. This study also did not aim to evaluate the resistance, especially to aspirin or other antiplatelet drugs such as adenosine diphosphate receptor inhibitors; however, additional platelet assays were not used.

\section{Strength of the study}

To the best of our knowledge, until now, there is no previous studies that have investigated the difference in dh-TxB2 in male and female ACS patients after PTCA and its associations with long-term MACE. The strengths of this present study include a very low dropout of patients $(2.4 \%)$ during follow-ups as patients were counseled and interviewed timely. We maintained strict inclusion criteria to observe the thromboxane concentration more precisely and make the study more impactful. We excluded patients with conditions like heart failure, severe liver dysfunction, patients with renal insufficiency, history of anemia or low hemoglobin, and any blood diathesis, which can influence dh-TxB2 levels or alter the platelet functions and outcomes. We recruited only patients who had developed ACS for the first time and underwent PCI with DES and who were started on antiplatelet therapy or any other antithrombotic therapy for the first time. In this cohort of 192 patients, we carried out the most sensitive quantitative assay at two time points, baseline and 6 months follow-up, which enabled us to conclude the dh-TxB2 value as a predictive biomarker. Keeping the real scenario in mind, we planned our study objectives and time of assay. Therefore, we carried out thromboxane assay within 24 hours in post-angioplasty patients who were loaded with antiplatelet drugs to imitate more of a "real-life scenario."

\section{CONCLUSION}

In this study, the dh-TxB2 level was significantly higher in females than males at baseline but not at 6 months. Overall, MACE was not significantly different between genders even when the dh-TxB2 levels were high. Whether this translates into a higher MACE rate during follow-up needs to be studied further.

\section{CONFLICT OF INTEREST}

Dr. Tom Devasia has received research grants from Novartis, India. Dr. Ajit Singh and Dr. Ganesh Paramasivam are working as co-investigator in clinical trials funded by Novartis, India. All the above-mentioned declarations have no impact on the current study. Other authors do not declare any conflict of interest.

\section{FUNDING}

No external/industry funds were received for the present study.

\section{AVAILABILITY OF DATA AND MATERIAL} is needed.

Data could be provided on the request of the editor if it

\section{AUTHORS' CONTRIBUTIONS}

All the authors are eligible to be an author as per the international committee of medical journal editors (ICMJE) requirements/guidelines.

\section{REFERENCES}

Ames PR, Batuca JR, Muncy IJ, De La Torre IG, Pascoe-Gonzales S, Guyer K, Matsuura E, Lopez LR. Aspirin insensitive thromboxane generation is associated with oxidative stress in type 2 diabetes mellitus. Thromb Res, 2012; 130(3):350-4.

Badimon L, Padró T, Vilahur G. Atherosclerosis, platelets and thrombosis in acute ischaemic heart disease. Eur Heart J Acute Cardiovasc Care, 2012; 1(1):60-74. 
Cipollone F, Ciabattoni G, Patrignani P, Pasquale M, Di Gregorio D, Bucciarelli T, Davì G, Cuccurullo F, Patrono C. Oxidant stress and aspirin-insensitive thromboxane biosynthesis in severe unstable angina. Circulation, 2000; 102(9):1007-13.

Eikelboom JW. Clopidogrel for high Atherothrombotic Risk and Ischemic Stabilization, Management and Avoidance (CHARISMA) investigators. Incomplete inhibition of thromboxane biosynthesis by acetylsalicylic acid: determinants and effect on cardiovascular risk. Circulation, 2008; 118:1705-12

Eikelboom JW, Hirsh J, Weitz JI, Johnston M, Yi Q, Yusuf S. Aspirin-resistant thromboxane biosynthesis and the risk of myocardial infarction, stroke, or cardiovascular death in patients at high risk for cardiovascular events. Circulation, 2002; 105(14):1650-5.

Gabrielsen A, Qiu H, Bäck M, Hamberg M, Hemdahl AL, Agardh H, Folkersen L, Swedenborg J, Hedin U, Paulsson-Berne G, Haeggström JZ. Thromboxane synthase expression and thromboxane A 2 production in the atherosclerotic lesion. J Mol Med, 2010; 88(8):795-806.

Gonçalves LH, Dusse LM, Fernandes AP, Gomes KB, Sóter MO, Alves MT, Rodrigues KF, Freitas FR, Komatsuzaki F, Sousa MO, Bosco AA. Urinary 11-dehydro-thromboxane B2 levels in type 2 diabetic patients before and during aspirin intake. Clin Chim Acta, 2011; 412(1516):1366-70.

Guo Y, Yin F, Fan C, Wang Z. Gender difference in clinical outcomes of the patients with coronary artery disease after percutaneous coronary intervention: a systematic review and meta-analysis. Medicine, 2018; 97(30):e11644.

Johansson S, Rosengren A, Young K, Jennings E. Mortality and morbidity trends after the first year in survivors of acute myocardial infarction: a systematic review. BMC Cardiovasc Disord, 2017; 17(1):53.

Josiah A, Farshid A. Gender is not a predictor of mortality or major adverse cardiovascular events in patients undergoing percutaneous coronary intervention for acute coronary syndromes. Heart Lung Circ, $2019 ; 28(5): 727-34$.

Kakouros N, Gluckman TJ, Conte JV, Kickler TS, Laws K, Barton BA, Rade JJ. Differential impact of serial measurement of nonplatelet thromboxane generation on long-term outcome after cardiac surgery. J Am Heart Assoc, 2017; 6(11):e007486.

Kakouros N, Nazarian SM, Stadler PB, Kickler TS, Rade JJ Risk factors for nonplatelet thromboxane generation after coronary artery bypass graft surgery. J Am Heart Assoc, 2016; 5(3):e002615.

Kareem H, Shetty PN, Devasia T, Karkala YR, Paramasivam G, Guddattu V, Singh A, Chauhan S. Impact of socioeconomic status on adverse cardiac events after coronary angioplasty: a cohort study. Heart Asia, 2018; 10(2):e010960.

Kurlansky P, Herbert M, Prince S, Mack M. Coronary bypass versus percutaneous intervention: sex matters. The impact of gender on long-term outcomes of coronary revascularization. Eur J Cardiothorac Surg, 2017; 51(3):554-61.
Liu H, Xu Z, Sun C, Chen Q, Bao N, Chen W, Zhou Z, Wang $\mathrm{X}$, Zheng Z. Perioperative urinary thromboxane metabolites and outcome of coronary artery bypass grafting: a nested case-control study. BMJ Open, 2018; 8(8):e021219.

Lopez LR, Guyer KE, De La Torre IG, Pitts KR, Matsuura E, Ames PR. Platelet thromboxane (11-dehydro-Thromboxane B2) and aspirin response in patients with diabetes and coronary artery disease. World $\mathrm{J}$ Diabetes, 2014; 5(2):115.

McCullough PA, Vasudevan A, Sathyamoorthy M, Schussler JM, Velasco CE, Lopez LR, Swift C, Peterson M, Bennett-Firmin J, Schiffmann R, Bottiglieri T. Urinary 11-dehydro-thromboxane B2 and mortality in patients with stable coronary artery disease. Am J Cardiol, 2017; 119(7):972-7.

Pendyala LK, Torguson R, Loh JP, Kitabata H, Badr S, Dvir D, Barbash IM, Satler LF, Pichard AD, Waksman R. Comparison of adverse outcomes after contemporary percutaneous coronary intervention in women versus men with acute coronary syndrome. Am J Cardiol, 2013; 111(8):1092-8.

Rao U, Buchanan GL, Hoye A. Outcomes after percutaneous coronary intervention in women: are there differences when compared with men? Interv Cardiol Rev, 2019; 14(2):70.

Smith JN, Negrelli JM, Manek MB, Hawes EM, Viera AJ. Diagnosis and management of acute coronary syndrome: an evidence-based update. J Am Board Fam Med, 2015; 28(2):283-93.

Szczeklik W, Stodółkiewicz E, Rzeszutko M, Tomala M, Chrustowicz A, Żmudka K, Sanak M. Urinary 11-dehydro-thromboxane B2 as a predictor of acute myocardial infarction outcomes: results of leukotrienes and thromboxane in myocardial infarction (LTIMI) study. J Am Heart Assoc, 2016; 5(8):e003702.

Vasudevan A, Tecson KM, Bennett-Firmin J, Bottiglieri T, Lopez LR, Peterson M, Sathyamoorthy M, Schiffmann R, Schussler JM, Swift C, Velasco CE. Prognostic value of urinary 11-dehydro-thromboxane B2 for mortality: a cohort study of stable coronary artery disease patients treated with aspirin. Catheter Cardiovasc Interv, 2018; 92(4):653-8.

Wang N, Vendrov KC, Simmons BP, Schuck RN, Stouffer GA, Lee CR. Urinary 11-dehydro-thromboxane B2 levels are associated with vascular inflammation and prognosis in atherosclerotic cardiovascular disease. Prostaglandins Other Lipid Mediat, 2018; 134:24-31.

How to cite this article:

Chauhan S, Singh A, Karkala YR, Devasia T, Kareem H, Uppunda D, Shetty PN, Paramasivam G. Gender-specific 11-dehydro-thromboxane B2 levels in acute coronary syndrome and its association with clinical outcomes. J Appl Pharm Sci, 2020; 10(11):010-017. 